\title{
Liquid crystal spherical caps in magnetic fields
}

\author{
P. Salamon $\odot,{ }^{1}$ Z. Karaszi, ${ }^{2}$ V. Kenderesi, ${ }^{1}$ Á. Buka, ${ }^{1, *}$ and A. Jákli ${ }^{1,2,3, \dagger}$ \\ ${ }^{1}$ Institute for Solid State Physics and Optics, Wigner Research Centre for Physics, P.O. Box 49, Budapest H-1525, Hungary \\ ${ }^{2}$ Chemical Physics Interdisciplinary Program, Advanced Materials and Liquid Crystal Institute, Kent State University, Kent, Ohio 44242, USA \\ ${ }^{3}$ Department of Physics, Kent State University, Kent, Ohio 44242, USA
}

(Received 18 March 2020; accepted 8 May 2020; published 2 June 2020)

\begin{abstract}
Spherical cap shaped liquid crystal drops have potential for use in tunable multifocal optical lenses. To achieve this, one needs to understand the liquid crystal director structure in various external fields. We present detailed experimental and theoretical studies of the director structure in magnetic fields of less than $1 \mathrm{~mm}$ diameter nematic liquid crystal drops on glass surfaces $(x-y$ plane), forming spherical cap shape with average molecular directions initially perpendicular to the boundaries. We show that at sufficiently high magnetic fields a Néel wall-type metastable inversion wall forms in the middle of the drop and then moves outward when the field is applied in $x$ direction. Applying fields above a critical angle between the plate of the spherical cap and the magnetic field, a uniform director structure forms. Drops with uniform director structure can be used as tunable optical lenses, where the focal length can be controlled by light polarization, viewing angle, magnetic, or electric fields. We also propose a theory that explains the texture variation in small magnetic fields and accounts for the formation and motion of the inversion wall. The theory explains the physical origin of the movement and gives the right magnitude of the speed at the first 10-15 min of the motion.
\end{abstract}

DOI: 10.1103/PhysRevResearch.2.023261

\section{INTRODUCTION}

Optical lenses with spherical cap shapes have been polished and carefully fabricated since $750 \mathrm{BC}$ and became essential for telescopes, microscopy, and photography. Tunable liquid microlenses capable of adjusting the focal length and position have been discovered only at the beginning of the 21 st century [1-5].

Optical lenses made from birefringent materials (anisotropic materials characterized with two different refractive indices) also have a wide range of applications, including image processing [6], common-path profilometry systems [7,8], multifocal intraocular elements [9], and compact-disk readers [10]. Traditionally, these birefringent lenses are made from crystals such as quartz, which provides excellent uniformity and transparency in the UV-visible range, but it is not tunable and requires precise fabrication. To tune optical axis and effective birefringence, nematic liquid crystal (NLC) is an excellent choice as it can be easily switched with magnetic or electric fields [11]. The latter property is utilized in the multibillion-dollar liquid crystal display (LCD) technology marketed these days as LED and QLED displays.

\footnotetext{
*Corresponding author: buka.agnes@wigner.mta.hu

†Corresponding author: ajakli@kent.edu
}

Published by the American Physical Society under the terms of the Creative Commons Attribution 4.0 International license. Further distribution of this work must maintain attribution to the author(s) and the published article's title, journal citation, and DOI.
Concepts of switchable LC microlenses can be classified to two main categories [12]. In the first one, the LC films have uniform thickness $h$ and the director field, i.e., optic axis, is spatially varied by electric fields applied across the film substrates. This leads to a gradient of effective refractive index $n_{\text {eff }}$ that results in a bent optical path [13-18]. In the second class, glass lenses are blended with switchable liquid crystals that enable changing either the surrounding refractive index of a convex glass lens [19-23] or by switching the polarization of the incoming light with an LC polarization rotator placed in front of a polymerized birefringent LC lens [24]. Recently, liquid crystals with tunable lens shapes have also been demonstrated either by using microfluidic techniques $[25,26]$, by chiral additive [27], or light [28].

However, to the best of our knowledge, so far, no studies described the director structure of lens-shaped fluid liquid crystals in external fields. This is a specially complicated case, as not only the film thickness varies, but also because the liquid crystal might be imposed to two different boundary conditions at the flat LC-solid and at the curved LC-isotropic medium interfaces [29]. In addition, the elastic anisotropy and the external fields also complicate the description. For example, when the LC director is aligned uniformly along the flat surface (planar anchoring), a "bipolar" state may form in which the director field has two surface point defects.

In this paper we describe experimental and theoretical studies of the director structure of lens-shaped nematic liquid crystal drops in various magnetic fields. The LC drops with free surface are placed on flat transparent substrates in the $x-y$ plane that align the director perpendicular to the plane. As the director is also perpendicular to the air-LC interface, at small contact angles, the LC lens is quasi-isotropic with a focal length independent of the polarization orientation, but 


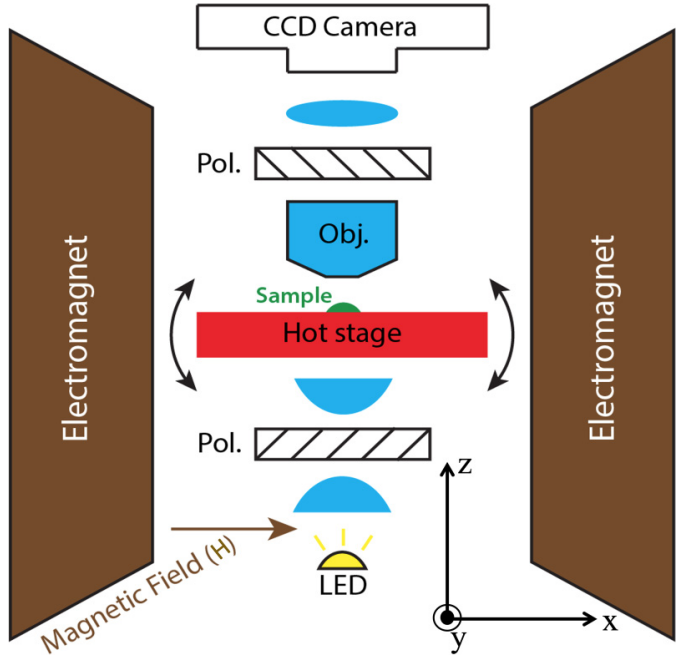

FIG. 1. Schematics of the custom-made polarizing optical microscopy system and a LC drop in a heat stage placed between the poles of an electromagnet.

dependent on the viewing angle. We show that, even under low magnetic fields, the director structure can be distorted and gradually leading to a defect wall that moves toward the periphery. We will also show that eventually, or by varying the direction of the magnetic field, the LC inside the drop can be aligned uniformly parallel to the magnetic field. The uniform director structure of such a drop may either be fixed by a polymer network and the focal length can be switched by a polarization rotator element or the focal length can be tuned by magnetic field.

\section{EXPERIMENTAL SETUP}

A custom designed polarizing optical microscope made of nonmagnetic materials was used that was placed between the poles of an electromagnet. The schematics of the setup can be seen in Fig. 1. The optical train of the microscope was vertical $(z)$, the drop was illuminated either by white or $633 \mathrm{~nm}$ wavelength monochromatic light, and the homogeneous magnetic field $(H)$ could be applied horizontally in $x$ direction; thus objects in the $x-y$ plane could be observed. The sample was placed between crossed polarizers that are rotated $\pm 45^{\circ}$ with respect to the $x$ axis. The temperature of the sample holder could be controlled with better than $\pm 0.1^{\circ} \mathrm{C}$ precision. The hot stage was rotatable around the $y$ axis. The angle of the base plate with respect to the $x$ direction is denoted by $\beta$. The maximum magnetic field slightly above $10^{4}$ Oe could be achieved. Micrographs could be recorded with an Optronics Microfire color CCD camera.

All measurements were done using the standard liquid crystal 4'-octyl-4-biphenylcarbonitrile (8CB) in the nematic phase at $39.8^{\circ} \mathrm{C}, 0.5^{\circ} \mathrm{C}$ below the isotropic-nematic phase transition. Liquid crystal droplets were put in the isotropic phase on glass substrates coated with JALS204 (a type of polyimide from JSR Co., Japan) polymer layers providing molecular orientation perpendicular (homeotropic) to the surface.

\section{RESULTS}

Just as isotropic liquids, fluid liquid crystals can also form spherical cap (lens) shapes by placing them on a flat substrate when the diameter of the drop is smaller than the capillary length $L_{c}=\sqrt{\gamma / \rho g}$ [30], which for the studied 8CB is about $1.8 \mathrm{~mm}$. With a good approximation, their contact angle $\theta$ can be given by Young's equation [31]: $\cos \theta=\left(\gamma_{S A}-\gamma_{S L}\right) / \gamma_{L A}$, where $\gamma_{S A}, \gamma_{S L}$, and $\gamma_{L A}$ are the surface tensions at the solidair, solid-liquid, and liquid-air interfaces, respectively. For liquid crystals where the director is perpendicular to the solid substrate, $\gamma_{S L}$ does not depend on the lateral direction, and the shape of the projection of the drop on the substrate is circular. For the JALS204 homeotropic surface alignment coating we used, the contact angle was found to be $\theta \approx 20^{\circ}$. Without external fields, such a small angle results in a fairly dark texture between crossed polarizers [see Fig. 2(a)] with four roughly equal intensity brighter spots situated symmetrically along the center lines at $x$ and $y$ directions [see texture in the inset of Fig. 2(a) taken with longer exposure time]. For the majority of liquid crystals, except for dimers [32], and a few other examples [33,34], the director is perpendicular to the air and the director structure is smooth, except for a defect ring around the drop [see sketch of the director structure in the $x-z$ plane in Fig. 2(a)]. Applying a field along $x$, it breaks the cylindrical symmetry of the droplet and gradually realigns the director along $x$.

The texture starts changing with the field in a way that the two spots on the $y$ axis (where $x=0$ ) become weaker, while those on the $x$ axis get brighter. This can be understood by considering that at $y \neq 0$ the director has $y$ component, which increases with $y$. A magnetic field applied along the $x$ direction leads to an increase of the $x$ component of the director while reducing the $y$ and $z$ components. As the twist angle inside the drop reaches $45^{\circ}$, the projection of the director to the base plate becomes parallel to one of the polarizers and the transmitted intensity vanishes. As seen in Fig. 2(b) for a 490 $\mu$ m diameter drop, at $H \sim 1 \mathrm{kOe}$ the spots on the $y$ axis almost disappear, while the other two spots brighten. The positions of these spots are approaching the center at increasing fields, marking the places where the optical paths $p$ have maxima. Above $H_{1} \sim 1.3 \mathrm{kOe}$, an increasing number of fringes appear around colored spots [see Fig. 2(c)]. In this stage, the director becomes almost parallel to the magnetic field inside the drop except for the center line where it stays homeotropic, and near the boundaries within a range determined by the magnetic coherence length that decreases at increasing fields (see Discussion). Increasing the fields further, the fringes become half circles separated by a central defect wall that spans between the drop boundaries perpendicular to the magnetic field [Fig. 2(d)]. At constant fields [10 kOe for the case shown in Fig. 2(e)] the defect wall moves gradually away the center line. Note that the direction of the movement (left or right) depends on the sign of the angle between the base plate and the magnetic field. Even less than $0.01^{\circ}$ inversion in the angle can cause different movement directions. At constant tilt the wall always moves in the same direction. Finally, after about $100 \mathrm{~min}$, the defect wall reaches the edge and stops [Fig. 2(f)]. In this stage the fringes are almost circular, and the director structure is uniformly parallel to the magnetic field. Note that, 


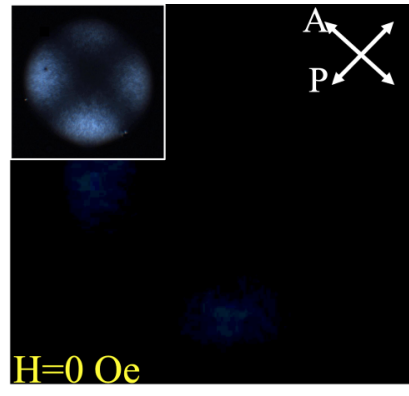

(a)
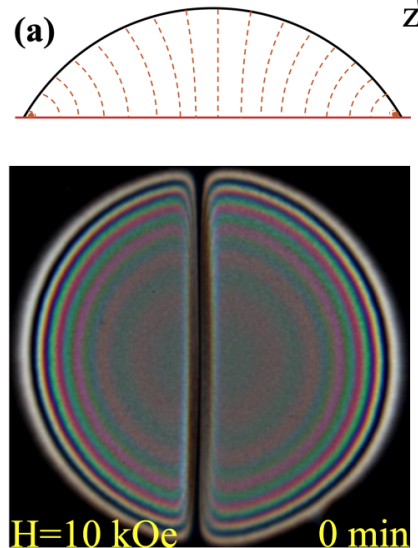

(d)

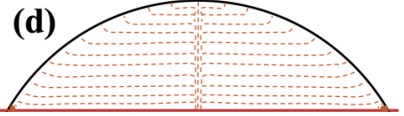

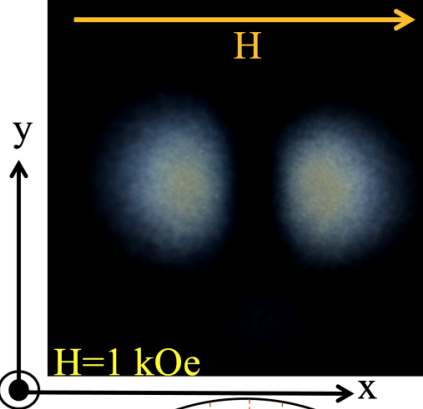

(b)
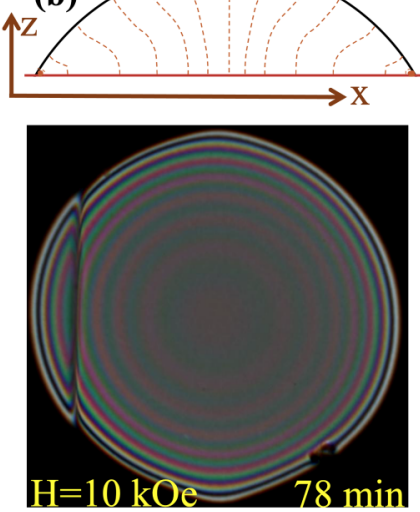

(e)

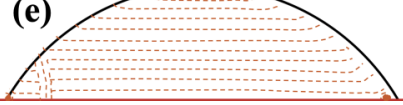

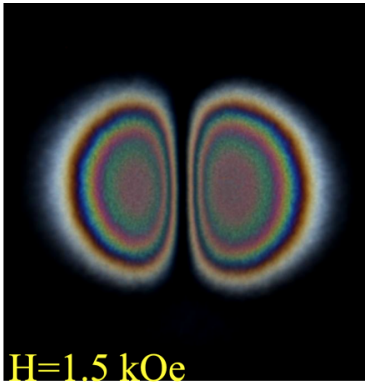

(c)
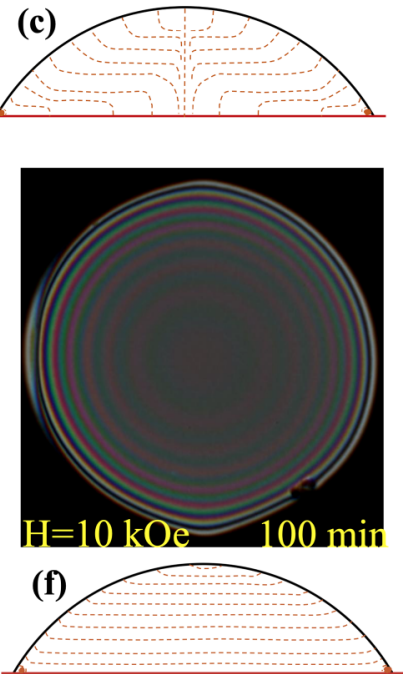

FIG. 2. Top view of textures between crossed polarizers at $\pm 45^{\circ}$ with respect to the magnetic field of a $490 \mu \mathrm{m}$ diameter drop at $39.8^{\circ} \mathrm{C}$ illuminated by white light (top images) and the approximate director structures at side view (bottom drawings) at different magnetic fields (a)-(c) and at different times (d)-(f). The inset in (a) shows the same drop with a higher exposure.

except for a small bulging in the $y$ direction, the base of the drop remains circular, indicating that the alignment at the surface is still perpendicular to the substrate, i.e., $\gamma_{S L}$ has only one value corresponding to that of the homeotropic alignment. Such a circular shape provides ideal birefringent lens conditions. Cooling the sample to the SmA phase, while the $10 \mathrm{kOe}$ field is maintained, the alignment reverts to homeotropic as in Fig. 2(a), proving that the anchoring is unbroken even at 10 $\mathrm{kOe}$ fields.

The magnetic field dependence (up to $1 \mathrm{kOe}$ ) of the average peak position and the $x$ dependence of the measured transmitted monochromatic light intensity at $y=0$ are shown in Fig. 3 for a $912 \mu \mathrm{m}$ dimeter drop. At increasing fields, the peak positions shift toward the center without a sharp threshold as it will be discussed later.

At fields above $1 \mathrm{kOe}$, the director rearrangement has been characterized by counting the number of interference fringes seen in Fig. 2(c). The number of the dark and bright fringes when a $912 \mu \mathrm{m}$ diameter sample is viewed through a red filter $(\lambda=633 \mathrm{~nm})$ is shown at increasing (red squares) and decreasing (blue dots) fields in the main pane of Fig. 4. After the first fringe appears at $\sim 0.9 \mathrm{kOe}$, the number of fringes increases strongly up to $1.5 \mathrm{kOe}$, then gradually saturates. Keeping in mind that there is a director distortion even below the appearance of the first fringe (see Fig. 3 and indicated by dotted line in Fig. 4), the behavior is typical for a Frederickstype transition with a pretilt. On decreasing fields, there is a small hysteresis that might be related to dynamics or some impurities. The inset of the figure shows the top view of the drop at $6 \mathrm{kOe}$ with 22 fringes and illustrating the way the fringes counted. In this particular drop there was a dust particle in the middle of the drop that pinned the movement of

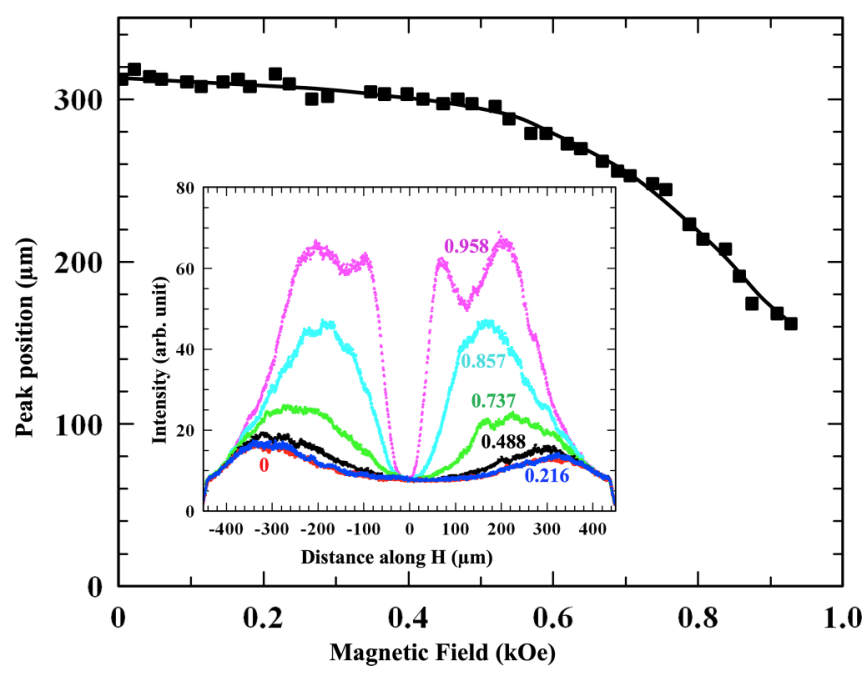

FIG. 3. Magnetic field dependence of the peak position from the center along the magnetic field of a $912 \mu \mathrm{m}$ diameter droplet at $39.8^{\circ} \mathrm{C}$. The inset shows the $x$ dependence of the intensity along a center line parallel to the magnetic field at various magnetic fields in kOe units. 


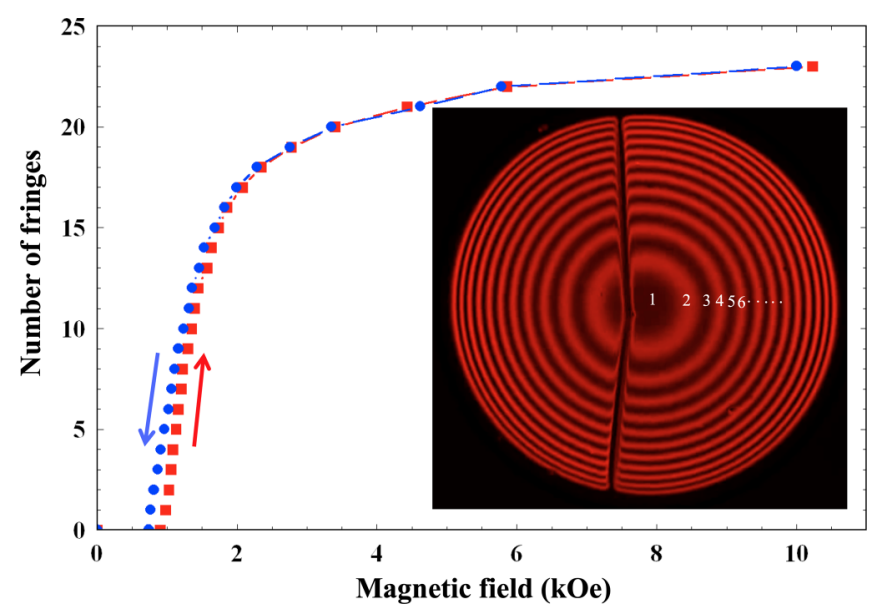

FIG. 4. Main pane: number of measured bright and dark fringes as the function of the magnetic field of a $912 \mu \mathrm{m}$ diameter spherical cap at $39.8^{\circ} \mathrm{C}$. The inset shows a drop with a dust particle in the center at $6 \mathrm{kOe}$ field with 22 fringes and illustrating the way the fringes are counted.

the defect wall. In the absence of any visible dust, the defect wall position in the middle becomes unstable and it is always moving toward one side.

The movement of the defect wall seen in Figs. 2(d)-2(f) is depicted in Fig. 5 for a $490 \mu \mathrm{m}$ diameter drop, where we plotted the time dependence of the position of the defect wall for different angles $-3^{\circ}<\beta<+3^{\circ}$ between the base of the drop and the magnetic field. In these experiments the magnetic field was ramped up to $10 \mathrm{kOe}$ in $100 \mathrm{~s}$, and the time dependence of the position was measured choosing $t=0$ when the field reached $10 \mathrm{kOe}$. For $\beta \cong 0$, the wall forms nearly at the center of the drop $[x(0) \approx 0]$, while for $\beta \neq 0,|x(0)|>0$ and moves to the same direction. Tilting the sample to the opposite direction the sign of $x(0)$ and $x(t)$ changes, too. In Fig. 5 we depicted $x(t)$ for four different

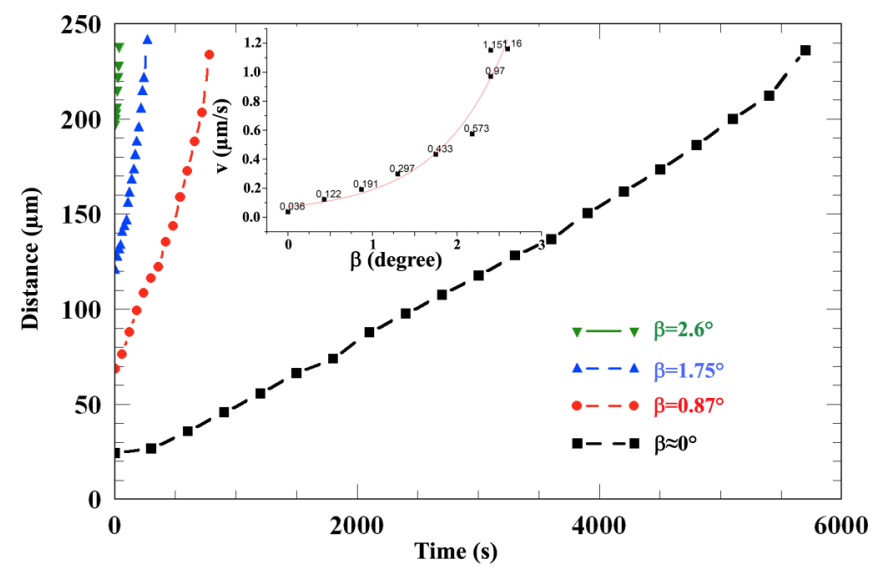

FIG. 5. Time dependence of the position of the defect wall for a $490 \mu \mathrm{m}$ diameter drop at $39.8^{\circ} \mathrm{C}$ at different angles between the sample plate and the magnetic field. The inset shows the average speed as the function of angle with fit line corresponding to an exponential function.

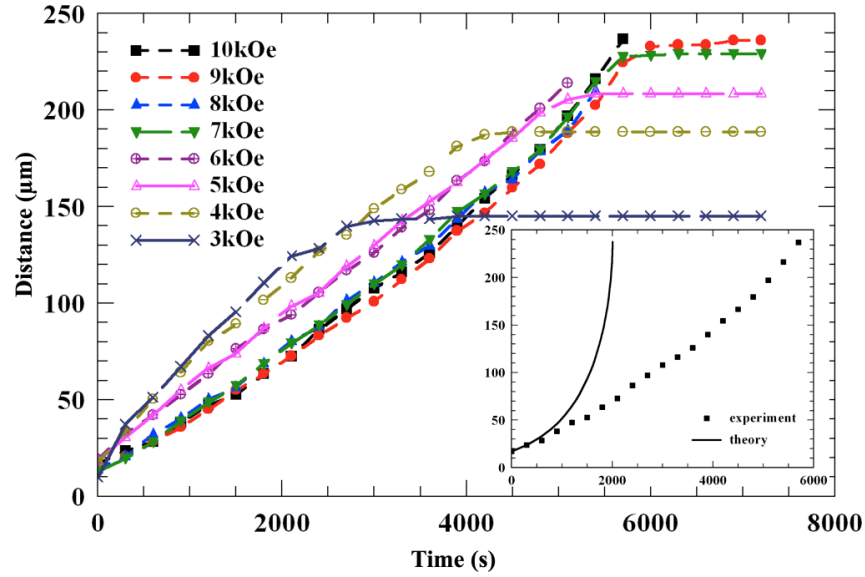

FIG. 6. Time dependence of the position of the defect wall for $490 \mu \mathrm{m}$ diameter drop at $39.8^{\circ} \mathrm{C}$ at different magnetic fields. The inset shows the time dependences of the displacement of the defect wall at $10 \mathrm{kOe}$ as measured and as calculated by the theory to be discussed in Sec. IV C.

tilt angles. It can be seen that $x(t)$ is almost proportional to the time, with $d x(t) / d t$ increasing slightly at $t>5000 \mathrm{~s}$. The movement is slowest for $\beta \cong 0$ and increases quickly at increasing angles. The angle dependence of the average speed is shown in the inset. One can see that the speed increases exponentially from $v \approx 0.036 \mu \mathrm{m} / \mathrm{s}$ at $\beta \approx 0^{\circ}$ to $1.15 \mu \mathrm{m} / \mathrm{s}$ for $\beta=2.6^{\circ}$. For $\beta=2.6^{\circ}$, the wall forms near to the edge of the droplet (about $200 \mu \mathrm{m}$ from the center). At larger tilt angles, there is no defect wall formation.

The time dependences of the distance of the defect walls from the middle were also measured at different constant magnetic fields for $\beta \approx 0$ and are plotted in Fig. 6. In the 7-10 kOe range, the speed of the wall is essentially the same up to about $5500 \mathrm{~s}$, when the wall reaches the edge of the droplet for $10 \mathrm{kOe}$ [shown in Fig. 2(f)], but stops at $225 \mu \mathrm{m}$ for 7, 8, and $9 \mathrm{kOe}$. Interestingly, decreasing the fields below $7 \mathrm{kOe}$, the initial speed of the wall increases, but the wall stops at smaller distances: $\sim 120 \mu \mathrm{m}$ for $3 \mathrm{kOe}, \sim 180 \mu \mathrm{m}$ for $4 \mathrm{kOe}$, and $\sim 210 \mu \mathrm{m}$ for $5 \mathrm{kOe}$.

The inset shows the time dependences of the displacement of the defect wall at $10 \mathrm{kOe}$ as measured, and as calculated by the theory to be discussed in Sec. IV C.

\section{DISCUSSION}

To model the behavior of the pattern in different magnetic fields, in Fig. 7 we sketch the geometry of the spherical cap and the director structure of the liquid crystal in different cross sections and different conditions.

\section{A. Transmittance in zero magnetic field}

From Fig. 7(a) we can write that $r_{o} \cos [\alpha(x)]=r_{o}-h_{o}+$ $h(x)$ and $\sin [\alpha(x)]=x / r_{o}$. Since $\alpha \leqslant \theta_{o}=20^{\circ}$, with good approximation $h(x) \simeq h_{o}-x^{2} / r_{o}$ and $\alpha(x) \simeq x / r_{o}$. 

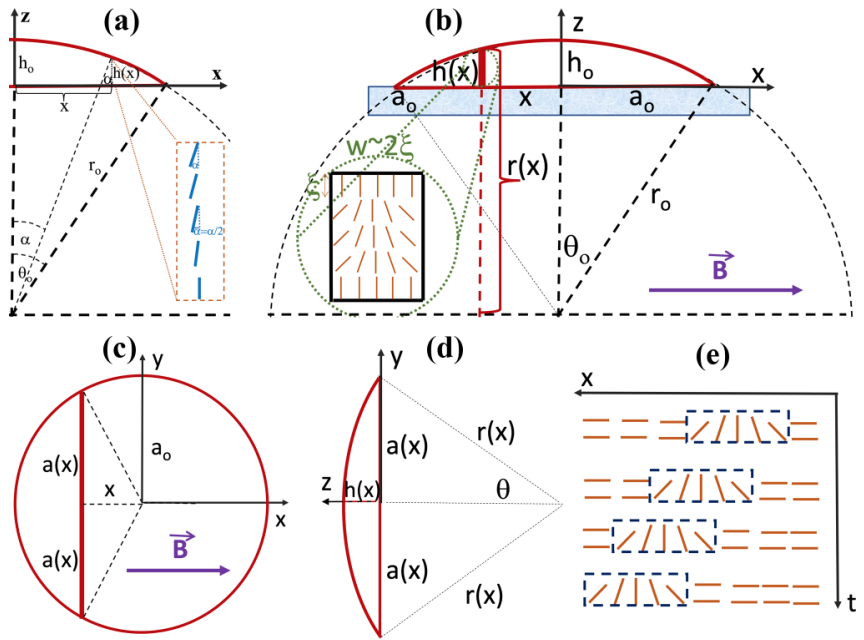

FIG. 7. Cross sections of the liquid crystal spherical cap in different planes and different states. (a) $x$ - $z$ plane at the center line of the drop at zero magnetic field, with the director distribution magnified (blue) along $z$. (b) $x-z$ plane showing the geometry of the defect wall (directors in orange, magnifier in green) at higher magnetic fields. Light blue shaded area indicates the glass plate; (c) $x-y$ plane showing the geometric relation between $a(x)$ and $a_{0}$. (d) $y-z$ plane showing the relation between $h(x)$ and $a(x)$; (e) $x-t$ plane showing the time dependence of the defect wall's position (blue dashed box) in $x$ direction.

The $x$ dependence of the transmittance $T(x)$ between crossed polarizers can be written as

$$
T(x)=\sin ^{2}(2 \Phi) \sin ^{2}\left(\frac{\pi}{\lambda} \int_{0}^{h(x)} \Delta n_{\mathrm{eff}}(x, z) d z\right) .
$$

Here $\Delta n_{\text {eff }}$ is the effective birefringence and $\Phi$ is the angle between director and the polarization of the incoming light of wavelength $\lambda$.

In the cross section along $x$, the projection of the director in the plane of the light polarization makes $\Phi= \pm 45^{\circ}$ with the polarizers; therefore, the first term in Eq. (1) is equal to one and the transmittance is determined solely by the retardation term. It is also well known [11] that $\Delta n_{\text {eff }}$ depends on the angle $\alpha(x, z)$ between the transmitted light ( $z$ direction) and the director as

$$
\Delta n_{\mathrm{eff}}(x, z)=\frac{n_{e}}{\sqrt{1+v \cos ^{2} \alpha(x, z)}}-n_{o},
$$

where $n_{o}\left(n_{e}\right)$ is the ordinary (extraordinary) refractive index and $v=\left(n_{e}^{2}-n_{o}^{2}\right) / n_{o}^{2}$. Without external field, the $z$ dependence of the director linearly increases from zero at the bottom of the cell up to $\alpha(x)$ at the top [see Fig. 2(a)], and the integral in Eq. (1) can be replaced with the average value $\Delta \bar{n}=\Delta n(\alpha / 2)$.

We therefore get that

$$
T(x)=\sin ^{2}\left(\frac{\pi \Delta n[\alpha(x) / 2] h(x)}{\lambda}\right) .
$$

Taking $n_{o} \sim 1.55$ and $\Delta n_{\text {eff }} \sim 0.11$ at $0.5^{\circ} \mathrm{C}$ below the clearing point [35], we can estimate that $\Delta n[a(x) / 2]<$ $10^{-4}$. Taking into account that $a(x) / r(x)=\sin \theta(x)$ and $h(x)=r(x)-a_{o} / \tan \left(\theta_{o}\right)[$ see Fig. $7(\mathrm{~d})]$ and $a^{2}(x)=a_{o}^{2}-x^{2}$ [see Fig. 7(c)] we get that the height of the drop at the middle is $h_{o}=a_{o} / \sin \theta_{o}-a_{o} / \tan \theta_{o}$, which for the $2 a_{o}=$ $912 \mu \mathrm{m}$ diameter drop in Figs. 3 and 4 , and $\theta_{o}=20^{\circ}$ gives $h_{o}=84.5 \mu \mathrm{m}$. These values give that the phase shift is much smaller than 0.1 , explaining the fairly dark texture observed at zero magnetic field [see Fig. 2(a)].

\section{B. Transmittance in magnetic field}

In the case of a nematic fluid with positive diamagnetic susceptibility anisotropy, sandwiched between two plates parallel to the $x-y$ plane with homogeneous initial director field along $z$, one expects the transition occurs sharply above the Fredericks threshold field given in CGS units as

$$
H_{b_{o}}=\frac{\pi}{h_{o}} \sqrt{\frac{K_{33}}{\Delta \chi}} .
$$

Here the subscript $b$ stands for bend director deformation, $K_{33}$ is the bend elastic constant, $\Delta \chi$ is the diamagnetic susceptibility of the material, and $h_{o}$ is the distance between bounding plates. (Note that some expressions given here in CGS units [e.g., Eq. (4)] are also given in SI units in the Appendix.) As the height of the droplet decreases radially from the center, so the threshold field increases. Additionally, the transition is sharp only at $x=0$, where $\alpha(0)=0$, and becomes smoother at increasing distance from the center. Finally, for $y>0$, all three director deformations are involved and coupled to the magnetic field; therefore, a three-dimensional description should be considered for a precise description of the director deformation. Such a complicated theoretical description is out of the scope of the present paper and may be the subject of further theoretical studies. Below we present a simplified but rather effective model.

Looking at Fig. 4, where the peak position is plotted as the function of small magnetic field for a $2 a_{o}=912 \mu \mathrm{m}$ drop, we can see a smooth Fredericks transition at $H_{b o}(0) \sim 0.9 \mathrm{kOe}$. From Eq. (4), the above $H_{b o}$ and $h_{o}$ values give $K_{33} / \Delta \chi=5.8$. At the measured $39.8^{\circ} \mathrm{C} K_{33} \sim 0.4 \mu$ dyne [36], which gives $\Delta \chi \sim 7 \times 10^{-8}$ CGS. This agrees well with measurements close to the $N-I$ transition $[37,38]$.

Above $H_{b o}$, the position of the highest intensity spots is moving toward the center as shown in Fig. 3. As the optical path difference

$$
\gamma(x, H)=\int_{0}^{h(x)} \Delta n_{\mathrm{eff}}(x, z, H) d z
$$

reaches one, a dark spot appears on both sides of the center along the magnetic field, and as $\gamma(x)$ reaches to higher integers, an increasing number of dark and bright fringes appear, as shown in Fig. 2(c).

To calculate the magnetic field dependence of $\gamma(x, H)$, one has to solve the Euler-Lagrange equation

$$
\frac{d}{d z}\left(\frac{\partial f_{\text {tot }}}{\partial(\partial \Theta / \partial z)}\right)+\frac{d}{d x}\left(\frac{\partial f_{\text {tot }}}{\partial(\partial \Theta / \partial x)}\right)-\frac{\partial f_{\text {tot }}}{\partial \Theta(x, z)}=0 .
$$

Here $\Theta$ is the angle between the director and the $z$ axis and $f_{\text {tot }}=f_{\mathrm{el}}+f_{\text {mag }}$ is the free energy density taking into account the Frank elastic free energy density $f_{\text {el }}$ and the contribution from the magnetic interaction $f_{\text {mag }}$. Assuming no 
twist deformation in the $x-z$ cross section at the center of the $\operatorname{drop}(y=0)$, and taking into account that $0.5^{\circ} \mathrm{C}$ below the $I-N$ transition the splay $\left(K_{11}\right)$ and the bend $\left(K_{33}\right)$ elastic constant are nearly equal $\left(K_{11}=K_{33}=K \sim 0.3 \mu\right.$ dyne) [36], we get that

$$
f_{\mathrm{el}} \approx \frac{1}{2} K\left[\left(\frac{\partial \Theta}{\partial x}\right)^{2}+\left(\frac{\partial \Theta}{\partial z}\right)^{2}\right]
$$

The magnetic energy density can be written as [11] $f_{\text {mag }}=$ $f_{\text {mag }}^{o}-\frac{1}{2} \Delta \chi \sin ^{2} \Theta \cdot H^{2}$, where $f_{\text {mag }}^{o}$ does not depend on $\Theta$. With these, the Euler-Lagrange equation inside the drop becomes

$$
\xi^{2}\left(\frac{\partial^{2} \Theta}{\partial x^{2}}+\frac{\partial^{2} \Theta}{\partial z^{2}}\right)+\sin \Theta \cos \Theta=0 .
$$

Here $\xi=\sqrt{K / \Delta \chi} / H$ is the magnetic coherence length (length where the magnetic field rotates the director roughly by $45^{\circ}$ toward the field) [11]. For the general solution, this equation has to be solved numerically taking into account the boundary conditions: $\Theta=0$ at $z=0$ for $x>0$ and at $z \leqslant h_{o}$ for $x=0$, and $\Theta=\alpha(x)$ at $z=h(x)$. However, for $H>H_{s o}=H_{b o}=H_{o}$ (i.e., for $x>\xi$ ), we can assume that the director depends only on $z$ and its $z$ dependence can be obtained as [11]

$$
\begin{aligned}
& \Theta(z)=\pi / 2-2 \arctan [\exp (-z / \xi)] \text { for } z<h(x) / 2, x>\xi \\
& \Theta(z)=\pi / 2-2 \arctan [\exp \{-[h(x)-z] / \xi\}] \text { for } z>h(x) / 2, x>\xi
\end{aligned}
$$

For $H \geqslant 1 \mathrm{kOe}$, Eq. (9) will be valid for $x \geqslant 30 \mu \mathrm{m}$, i.e., for more than $92 \%$ of the area of a drop for $a_{o}=250 \mu \mathrm{m}$.

With these in mind, the transmittance in the center line along the magnetic field can be approximated as

$$
T(x)=\sin ^{2}\left[\frac{\pi}{\lambda} \int_{0}^{h(x)}\left(\frac{n_{e}}{\sqrt{1+v \cos ^{2} \Theta(z)}}-n_{o}\right) d z\right],
$$

where $\Theta(z)$ is taken from Eq. (9). The $k=24$ dark and bright fringes measured at $10 \mathrm{kOe}$ mean the optical path difference $\Delta n_{\text {eff }} \cdot h_{o} \sim k / 2 \cdot \lambda$. With the red filter $(\lambda=633 \mathrm{~nm})$ and $h_{o}=84.5 \mu \mathrm{m}$, we get that $\Delta n_{\mathrm{eff}}(10 \mathrm{kOe}) \approx 0.09$. This is slightly smaller than $\Delta n=n_{e}-n_{o}=0.11$ measured at $0.5^{\circ} \mathrm{C}$ below the $I-N$ transition [35] and is consistent with the observation that the homeotropic boundary condition was not broken at $10 \mathrm{kOe}$.

\section{Motion of the inversion wall}

As the magnetic field increases, the number of fringes increases, and their shape approaches half circles that are separated by an inversion wall with thickness twice the magnetic coherence length $\xi(H)=\sqrt{K_{33} / \Delta \chi} / H$ (see video in the Supplemental Material for a drop where snapshots are shown in Fig. 2) [39]. Although this inversion wall appears at (or close to) the center line of the droplet, it slowly starts moving toward the periphery along the magnetic field. We propose this is mainly because during this motion the defect area-and thus its energy-decreases. This provides an effective force that is balanced by the gradient of the energy dissipated during that motion.

In first approximation, we neglect the surface layer with magnetic coherence length $\xi\left(10^{4} \mathrm{Oe}\right) \sim 2.6 \mu \mathrm{m}$ in comparison to the height of the wall $h(x) \leqslant 43 \mu \mathrm{m}$ for the $490 \mu \mathrm{m}$ diameter drop. The cross section of the defect wall in the $x-z$ plane is sketched in Fig. 7(b). Looking at Fig. 7(d), we can express the area of the cross section of the defect wall at a distance $x$ from the center as

$$
A(x)=r^{2}(x) \theta(x)-a(x)[r(x)-h(x)],
$$

where $\theta(x)=\arcsin [a(x) / r(x)] \quad$ and $\quad h(x)=r(x)-$ $a_{o} / \tan \left(\theta_{o}\right)$. Substituting these expressions into Eq. (11), we can express $A(x)$ with variables $x, a_{o}$, and $\theta_{o}$ as

$$
\begin{aligned}
A(x)= & \arcsin \left[\frac{\sqrt{a_{o}^{2}-x^{2}}}{\sqrt{a_{o}^{2} \operatorname{Csc}^{2}\left(\theta_{o}\right)-x^{2}}}\right]\left[a_{o}^{2} \csc ^{2}\left(\theta_{o}\right)-x^{2}\right] \\
& -a_{o} \sqrt{a_{o}^{2}-x^{2}} \cot \left(\theta_{o}\right) .
\end{aligned}
$$

For small contact angles $\left(\theta_{o}=20^{\circ}\right)$, we can approximate $A(x)$ as

$$
A(x) \sim \frac{2\left(a_{o}^{2}-x^{2}\right)^{3 / 2} \theta_{o}}{3 a_{o}} .
$$

The energy of the defect wall is

$$
W_{D}=E \cdot A(x) .
$$

Here $E=2 H \sqrt{K_{33} \Delta \chi}$ is the energy per unit area of the defect wall in CGS units [40].

In this approximation at constant magnetic fields, the defect energy will be proportional to the area $A(x)$. Consequently, as $A(x)$ decreases with increasing $x$, the defect wall will be pushed toward the periphery.

The force that is pushing the wall sideways is the negative derivative of the energy with respect to $x$ :

$$
F(x)=-\frac{d W}{d x}=-E \frac{d A(x)}{d x} .
$$

As it can be seen in Fig. 7(e), the motion of the wall to keep its structure undistorted requires a director rotation by $180^{\circ}$ $(\pi)$ while the wall moves by its thickness $w$. This is similar to the flow of a cholesteric liquid crystal along the helix axis. It is called permeation and was described first by Helfrich [41]. The viscous torque density $\Gamma_{v}$ related to this rotation can be expressed as $\Gamma_{v}=\gamma_{1}(d \varphi / d t)$, where $\varphi$ is the angle of the director and $\gamma_{1}$ is the rotational viscosity. The rate of the director rotation is $d \varphi(x) / d t=\pi / T(x)$, where $T(x)$ is the time it takes to translate by a thickness of the wall with the 
speed $v(x)=w / T(x)$.. With these, we get that $d \varphi(x) / d t=$ $\pi v(x) / w$. The energy density dissipated by the rotation in unit time is $-\gamma_{1}[\pi v(x) / w]^{2}$. This dissipated energy density must be balanced by the energy density gain in unit time, which is the force density $F(x) /[A(x) \cdot w]$ :

$$
\gamma_{1}\left(\frac{\pi}{w} v(x)\right)^{2}=\frac{F(x) \cdot v(x)}{w \cdot A(x)} .
$$

From this, we can express $v(x)$ as

$$
v(x)=\frac{d x}{d t}=\frac{F(x) \cdot w}{A(x) \cdot \gamma_{1} \pi^{2}}=-\frac{E A^{\prime}(x) w}{A(x) \gamma_{1} \pi^{2}},
$$

where prime $\left({ }^{\prime}\right)$ means derivation by $x$. After separation of the variables, we can write

$$
-\frac{\gamma_{1} \pi^{2}}{E w} \int_{x_{o}}^{x} \frac{A(x)}{A^{\prime}(x)} d x=\int_{0}^{t} d t=t .
$$

Using $A(x)$ given in Eq. (12) the integral can be solved only numerically, while using the approximate expression of $A(x)$ of Eq. (13) we get

$$
a_{o}^{2} \ln \left(\frac{x}{x_{o}}\right)-\frac{x^{2}-x_{o}^{2}}{2}=\frac{6 H w \sqrt{K_{33} \Delta \chi}}{\gamma_{1} \pi^{2}} t .
$$

This expression apparently depends on the magnetic field $H$, the width of the defect wall $w$, the diamagnetic susceptibility anisotropy $\Delta \chi$, the bend elastic constant $K_{33}$, and the rotational viscosity coefficient $\gamma_{1}$. However, in the approximation that the thickness of the defect wall is $w \sim 2 \xi$, i.e., depends on the magnetic field as $w=2 \sqrt{K_{33} / \Delta \chi} / H$, where $\xi \ll$ $h(x)$, the magnetic field, and the diamagnetic susceptibility anisotropy drop out from the equation, and we get

$$
a_{o}^{2} \ln \left(\frac{x}{x_{o}}\right)-\frac{x^{2}-x_{o}^{2}}{2} \approx \frac{12 K_{33}}{\gamma_{1} \pi^{2}} t .
$$

This equation contains only the size of the drop $a_{o}$, two material parameters $K_{33}$ and $\gamma_{1}$, and predicts that $x(t)$ is independent of the magnetic field, which is indeed observed between $6 \mathrm{kOe}$ and $10 \mathrm{kOe}$ fields (see Fig. 6). Equation (20) does not hold at lower fields, where the magnetic coherence length becomes comparable to the height of the drop, thus Eq. (13) is not valid anymore.

Importantly, to solve Eq. (20) no fitting parameter is needed. Taking $a_{o}=245 \mu \mathrm{m}$, and published values of $\gamma_{1}=$ $50 \mathrm{cp}[42,43]$ and $K_{33}=2.8 \times 10^{-7}$ dyne [36] at the measured $0.5^{\circ} \mathrm{C}$ below the $I-N$ transition, we obtain the theoretical curve shown in the inset of Fig. 6. At the first 10-15 min of the movement this theory excellently agrees with the experimental results; later, however, the theoretical curve accelerates, while the real wall motion continues with almost constant speed. There are several possible reasons for this discrepancy.

(a) In obtaining Eq. (20), we used an approximate area given in Eq. (13) instead of the exact one in Eq. (12). Our numerical calculation, however, shows that-as expected-this does not make a large difference and still gives an accelerating curve.

(b) Since the tilt of the top surface $\alpha(x)$ with respect to the bottom plate increases with increasing $x$, the defect wall may become bent in order to stay perpendicular to both boundaries. This leads to a weaker $A(x)$ dependence than that given in Eq. (12), thus slowing down the motion. However, since $\alpha<20^{\circ}$, the correction is not expected to be sufficient to explain the difference between the experiments and theory for $t>30 \mathrm{~min}$.

(c) There might be impurities and defects that are dragged by the defect wall, thus slowing down the movement at later stages. In fact, we have observed that larger dust particles can even pin the defect wall. This is illustrated in the inset of Fig. 4, where a dust particle placed in the center completely halted the motion of the wall. After turning the field off and repeating the measurements several times, the measured time dependences reproduced, indicating that the distribution of the defects or impurities reproduces as well.

(d) Due to the no-slip boundary condition, the defect wall ends in two half defect lines at a distance $\xi$ from the wall [see Fig. 7(b)]. These defect lines are likely pinned at the peripheral defect ring, while they are dragged inside the drop by the defect wall. Consequently, the defect lines become increasingly stretched as the distance $x$ of the wall increases. Although this mechanism likely can explain the lack of acceleration, its theoretical description is out of the scope of this paper.

(e) Finally, the actual height of the defect $h_{d}=h(x)-2 \xi$ and the actual area of the defect wall $A_{d}(x)$ decreases faster than the calculated $A(x)$. Although this would provide even more acceleration at intermediate $x$ values, the Helfrich type dissipation mechanism fails at higher $x$, when the coherence length is comparable to the thickness of the material. Indeed, we find (see Fig. 6) that the wall stops at small $h(x)$ values. We assume that this happens when $h(x)=2 \xi$, i.e., when the wall shrinks to a defect line that cannot gain energy by further movement. Since $\xi$ is inversely proportional to the magnetic field, the distance $x$, where the $h(x)$ becomes twice of the coherence length, decreases qualitatively in accordance with the experimental results shown in Fig. 6. For example, at 3 kOe $\xi \sim 5 \mu \mathrm{m}$, i.e., it should stop at $h(x) \sim 10 \mu \mathrm{m}$. Since $x=h(x) \simeq h_{o}-x \sin \theta$, using $h_{o}=43 \mu \mathrm{m}$ and $\theta=20^{\circ}$ one gets that $x \sim 100 \mu \mathrm{m}$. This is close to the experimentally observed $x \sim 120 \mu \mathrm{m}$.

\section{CONCLUSIONS}

In this paper we described studies of the director structure of lens-shape nematic liquid crystals with perpendicular (homeotropic) boundary conditions in various external magnetic fields.

Experimentally, we observed that the optical path has two maxima in the center line along the magnetic field. On increasing fields, the positions of the maxima move closer to the center and lead to the formation of increasing number of fringes that eventually form half circles separated by an inversion wall at the center, perpendicular to the field. We find that the defect wall is unstable in the center line and moves toward the periphery along the magnetic field. Below $7 \mathrm{kOe}$ magnetic fields, the speed is larger for smaller fields, but the walls stop at smaller distances. In the $7-10 \mathrm{kOe}$ range, the speed is independent of the magnetic field.

Our theory can account for all major features of the experimental observations. (i) It can explain the number of fringes 
at the function of magnetic field, (ii) gives an explanation for the movement of the defect walls, (iii) gives an excellent description of the speed at the first 10-15 min of the defect wall motion without any fitting parameter, and (iv) it explains that the speed of the defect wall is independent of the magnitude of $H>7 \mathrm{kOe}$ magnetic fields. Although this theory deals only with magnetic interactions, it is straightforward to generalize to electric effects that might be more useful for tuning lenses.

For practical applications, the most promising observation is that the defect wall does not appear above a critical angle between the plate of the spherical cap and the magnetic field, thus giving rise to uniform director structure. After polymerization, or for materials with glass transition, such objects may be used to make optical lenses with a focal length that varies with the polarization and the direction of the light beam. Additionally, in its liquid form, the focal lengths can be tuned by magnetic field.

The experimental observations and their comparison to our theory imply a number of further research directions, such as investigating the role of impurities and defects on the movement of the defect wall, the study of the optical behavior of birefringent LC drops, or their tuning with magnetic and electric fields.

\section{ACKNOWLEDGMENTS}

This work was financially supported by the following grants: No. NSF DMR 1904167 and No. NKFIH FK125134. A.J. acknowledges useful discussions with Prof. J. Selinger and Prof. H. Yokoyama.

\section{APPENDIX}

Above we used quantities and equations in CGS units for simplicity. Here we give those related to our results in SI units as well.

The magnetic field $H=1$ Oe $(\mathrm{CGS})=(4 \pi)^{-1} \times$ $1000 \mathrm{~A} / \mathrm{m} \approx 79.58 \mathrm{~A} / \mathrm{m}$ (SI). For nonmagnetic materials, $H=1$ Oe (CGS) corresponds to $B=1 \mathrm{G}$ (CGS) magnetic inductance, which is $B=0.1 \mathrm{mT}$ in SI. In our studies, the maximum applied magnetic field was about $10 \mathrm{kOe}$, which corresponds to $B=1 \mathrm{~T}$. The Fredericks threshold field [Eq. (4)] in SI is $H_{b o}=\left(\pi / h_{o}\right) \sqrt{K_{33} / \mu_{o} \Delta \chi_{S I}}$, where $\mu_{o}=4 \pi \times 10^{-7} \mathrm{Vs} / \mathrm{Am}$ is the permeability of the vacuum and $\Delta \chi_{\text {SI }}$ is the diamagnetic susceptibility anisotropy in SI. The Frank elastic constants have units of force, which are dyne in CGS and $\mathrm{N}$ in SI; in our case $K_{33}=3 \times 10^{-7}$ dyne $=3 \mathrm{pN}$. The magnetic coherence length in SI is $\xi=(1 / H) \sqrt{K_{33} / \mu_{o} \Delta \chi_{S I}}$.
[1] B. Berge and J. Peseux, Eur. Phys. J. E 3, 159 (2000)

[2] L. Dong, A. K. Agarwal, D. J. Beebe, and H. Jiang, Nature (London) 442, 551 (2006).

[3] W. Xiao and S. Hardt, J. Micromech. Microeng. 20, 055032 (2010).

[4] T. Krupenkin, S. Yang, and P. Mach, Appl. Phys. Lett. 82, 316 (2003).

[5] N. Chronis, G. Liu, K.-H. Jeong, and L. Lee, Opt. Express 11, 2370 (2003).

[6] A. Ghosh and A. K. Chakraborty, Opt. Commun. 40, 329 (1982).

[7] M. J. Downs, W. H. McGivern, and H. J. Ferguson, Precis. Eng. 7, 211 (1985).

[8] J. P. Lesso, A. J. Duncan, W. Sibbett, and M. J. Padgett, Appl. Opt. 39, 592 (2000).

[9] W. Fiala, Optom. Vision. Sci. 69, 329 (1992).

[10] K. Kinnstatter, M. Ojima, and S. Yonezawa, Appl. Opt. 29, 4408 (1990).

[11] P.-G. de Gennes, The Physics of Liquid Crystals, 2nd ed. (Clarendon Press, Oxford, 1974).

[12] T. Scharf, Polarized Light in Liquid Crystals and Polymers (John Wiley \& Sons, Hoboken, NJ, 2007).

[13] S. Masuda, M. Honma, T. Nose, and S. Sato, Jpn. J. Appl. Phys. 36, 2765 (1997).

[14] M. Ye and S. Sato, Jpn. J. Appl. Phys. 40, 6012 (2001).

[15] M. Honma, T. Nose, and S. Sato, Jpn. J. Appl. Phys. 40, 1322 (2001).

[16] O. Pishnyak, S. Sato, and O. D. Lavrentovich, Appl. Opt. 45, 4576 (2006).

[17] S. Xu, Y. Li, Y. Liu, J. Sun, H. Ren, and S. T. Wu, Micromachines 5, 300 (2014).
[18] Y.-H. Lin, H.-S. Chen, and M.-S. Chen, Mol. Cryst. Liq. Cryst. 596, 12 (2014).

[19] J. S. Patel and K. Rastani, Opt. Lett. 16, 532 (1991).

[20] J.-H. Lee, J.-H. Beak, Y. Kim, Y.-J. Lee, J.-H. Kim, and C.-J. Yu, Opt. Express 22, 9081 (2014).

[21] L. G. Commander, S. E. Day, and D. R. Selviah, Opt. Commun. 177, 157 (2000).

[22] J. Kim, J. Kim, J.-H. Na, B. Lee, and S.-D. Lee, Opt. Express 22, 3316 (2014).

[23] H. Ren, J. R. Wu, Y. Fan, Y. Lin, and S. Wu, Opt. Lett. 30, 376 (2005).

[24] S. Sato, Jpn. J. Appl. Phys. 18, 1679 (1979).

[25] Q. Chen, T. Li, Z. Li, J. Long, and X. Zhang, Micromachines 9 , 97 (2018).

[26] D. Wee, S. H. Hwang, Y. S. Song, and J. R. Youn, Soft Matter 12, 3868 (2016).

[27] P. Popov, L. W. Honaker, M. Mirheydari, E. K. Mann, and A. Jákli, Sci. Rep. 7, 1603 (2017).

[28] Y. Li, Y. Liu, and D. Luo, J. Mater. Chem. C 7, 15166 (2019).

[29] V. K. Gupta and N. L. Abbott, Langmuir 15, 7213 (1999).

[30] Y. Yuan and T. R. Lee, in Surface Science Techniques, Springer Series in Surface Sciences, Vol. 51, edited by B. Bracco and G. Holst (Springer, Berlin, 2013), pp. 3-34.

[31] T. Young, Philos. Trans. R. Soc. Lond. 95, 65 (1805).

[32] J. Yoshioka, P. Salamon, D. A. Paterson, J. M. D. Storey, C. T. Imrie, A. Jákli, F. Araoka, and A. Buka, Soft Matter 15, 989 (2019).

[33] K. Nayani, P. Rai, N. Bao, H. Yu, M. Mavrikakis, R. J. Twieg, and N. L. Abbott, Adv. Mater. 30, 1706707 (2018).

[34] P. Chiarelli, S. Faetti, and L. Fronzoni, Lett. Nuovo Cimento 36, 60 (1983). 
[35] R. G. Horn, J. Phys. 39, 105 (1978).

[36] P. P. Karat and N. V. Madhusudana, Mol. Cryst. Liq. Cryst. 40, 239 (1976).

[37] H. Schad, G. Baur, and G. Meier, J. Chem. Phys. 71, 3174 (1979).

[38] A. Buka and W. H. de Jeu, J. Phys. 43, 361 (1982).

[39] See Supplemental Material at http://link.aps.org/supplemental/ 10.1103/PhysRevResearch.2.023261 for the motion of the de- fect wall of a $490 \mu \mathrm{m}$ diameter drop as the magnetic field increased to $10 \mathrm{kOe}$ in $100 \mathrm{~s}$, then kept constant.

[40] W. Helfrich, Phys. Rev. Lett. 21, 1518 (1968).

[41] W. Helfrich, Phys. Rev. Lett. 23, 372 (1969).

[42] P. Chattopadhyay and S. K. Roy, Mol. Cryst. Liq. Cryst. 237, 1 (1993).

[43] A. G. Chmielewski, Mol. Cryst. Liq. Cryst. 132, 339 (1986). 